\title{
ESTUDO SOBRE EFEITOS QUÂNTICOS E SIMETRIA
}

\author{
Bassem Youssef Makhoul Junior $^{1}$; Carlos Alberto De Lima Ribeiro ${ }^{2}$ \\ 1.Bolsista FAPESB, Graduando em Bacharelado em Física, Universidade Estadual de Feira de Santana, \\ bassem_ymj@hotmail.fr \\ 2. Orientador, Departamento de Física, Universidade Estadual de Feira de Santana, calr@uefs.br
}

PALAVRAS-CHAVE: Simetrias; Defeitos Topológicos; Efeitos Quânticos.

\section{INTRODUÇÃO}

O estudo das propriedades de um dado material, do ponto de vista de suas propriedades físicas, implica na utilização de ferramentas matemáticas aplicadas nesse tratamento ao analisarmos sob o ponto de vista quântico (WEBBER \& RICCI, 2007). Defeito Topológico é um tipo de defeito que é caracterizado por uma quebra de simetria contínua (KITTEL, 2005).

Inicialmente a Topologia começou sendo um ramo da matemática, que estuda os espaços topológicos, e considerados como uma extensão da geometria. Os Espaços Topológicos são estruturas que possibilitam a formalização de conceitos como convergência, convexidade e continuidade. Um problema clássico topológico, é o das 7 pontes de Königsberg. Em uma cidade, que era dividida em 3 partes pelo rio Pregel, dividido por 4 ilhas, era questionado, se possível, passar pelas 4 terras andando e atravessar sobre as 7 pontes da cidade de uma única vez. Leonhard Euler provou que isso era impossível, e principalmente, ele teve a ideia de associar gráficos com o problema, onde só era necessário fazer com que as somas dos vértices dos gráficos dessem um valor par. A Topologia só foi aplicada na física em 1960 por George Gamow, onde este, fez uma observação que teve muito destaque em suas primeiras obras, sendo que ambas revolucionaram a Física em no início do século XX: A teoria Quântica e a Relatividade (BOWMAN, 2008).

Algumas das principais aplicações da Topologia e os Defeitos Topológicos estão na Cosmologia, na Física da Matéria Condensada e nos estudos sobre Transições de Fases (GREINER \& MÜLLER, 1989). Quando aplicamos um conjunto de transformações a um sistema ou objeto, e depois destas transformações ele continua o mesmo, dizemos então que ele é simétrico. Um exemplo de simetria presente na rede cristalina é a de reflexão. $\mathrm{O}$ principal objetivo deste trabalho é ter uma noção conceitual sobre o que são efeitos quânticos e simetrias presentes em materiais de baixa dimensionalidade (HILL \& LEDERMAN, 2000). Para que seja possível aprofundar mais sobre modelos físicos com anéis quânticos e quebra de simetria de reversão temporal. Defeitos Topológicos tem sua origem em quebras de simetria. Como relacionar as simetrias pertencentes ao meio (cristalino) à aquelas simetrias presentes em Física Clássica? Como as simetrias na Mecânica Quântica se relacionam com a Mecânica Clássica?

\section{METODOLOGIA}

Leituras críticas de artigos e livros formaram a base do trabalho feito durante o ano de pesquisa, após os estudos e análises, foram feitos textos críticos sobre o dado conteúdo que posteriormente seriam discutidos com o orientador, visando solucionar duvidas e ideias que surgiram após a leitura do texto. Inicialmente foi feito o levantamento bibliográfico sobre o tema, visando entender como que surgiu Mecânica Quântica, bem como os defeitos topológicos, e o porquê de precisarem dela. Em seguida foram realizados estudos sobre 
algumas ferramentas matemáticas para a resolução de alguns problemas, como o do confinamento quântico de uma partícula em uma e duas dimensões. E por fim, foi estudado o que é uma simetria, as simetrias na Mecânica Clássica, e as principais diferenças entre simetrias na Mecânica Clássica para a Mecânica Quântica.

\section{RESULTADOS E DISCUSSÃO}

Nos sólidos cristalinos são encontrados diferentes tipos de defeitos. Existem 4 tipos de defeitos estruturais, o pontual, linear, planar e volumétrico. A lacuna é o defeito pontual mais comum e mais simples, que é a ausência de um ou mais átomos de sua posição original em uma estrutura cristalina. Existe também os defeitos intersticiais que é a presença de um átomo que não pertence a estrutura cristalina, podendo ocupar uma lacuna, à consequência disto é que vai causar uma distorção na estrutura cristalina devido ao desajuste causado pelo átomo. E o ultimo que é o defeito substicional, que ocorre quando um átomo muito maior que os outros está na célula unitária, e isso gera uma distorção, que introduz tensões, onde estas atuam como uma barreira ao movimento de deslocações (discordâncias) e aumenta a resistência do material. Defeitos lineares são defeitos que geram uma distorção da rede centrada em torno de uma linha de átomos, elas podem ser geradas também por condensação de lacunas ou por desajuste em sólidos. Os principais tipos de defeitos são as deslocações (discordâncias) em linha, cunha (arestas) e hélice espiral (parafuso). A combinação destas últimas gera a deslocações (discordância) mista (KITTEL, 2007).

Para encontrar um Defeito Topológico em um cristal, podemos utilizar o método do vetor de Burgers. O vetor de Burgers é a diferença entre a somatória de vetores que tinha antes e depois de acontecer algum tipo de defeito na estrutura cristalina. Por exemplo, se tiver uma estrutura cristalina em formato de um quadrado com quatro átomos e quatro vetores ligando um átomo a outro, e então acrescentar-se mais um átomo, ou seja, agora a estrutura tem 5 átomos formando um pentágono, o número total de vetores agora vai ser cinco, esse $5^{\circ}$ vetor, é o vetor de Burgers.

Defeitos planares são cristais que apresentam defeitos em duas dimensões. A superfície livre é a superfície entre o cristal e o meio que o circunda. Pelo fato dos átomos não estarem ligados ao número máximo de vizinhos, a energia dos átomos da superfície é menor que no interior. Defeitos volumétricos são cristais que apresentam defeitos em três dimensões. As principais características são a porosidade que a quantidade de poros numa substância e os precipitados que são aglomerados de partículas cuja a composição difere da matriz.

Foram utilizados alguns métodos matemáticos para o estudo do confinamento quântico de uma partícula em uma e duas dimensões, que foram a Normalização e a Probabilidade.

A normalização é afirmar que existe uma partícula em um dado espaço:

$$
\int_{-\infty}^{+\infty}|\varphi|^{2} d x=1
$$

A probabilidade se refere a probabilidade de encontrar um estado em um espaço determinado:

$$
\operatorname{Prob}_{a b}=\int_{a}^{b}|\varphi|^{2} d x
$$

Sendo "a" e "b" os limites do meu sistema.

Tendo essas ferramentas matemáticas, podemos investigar o confinamento quântico de uma partícula e achar a energia associada à função de onda. $\mathrm{O}$ problema do confinamento quântico de um partícula consiste em uma partícula presa numa região onde dizemos que é limitada por uma energia potencial que tende ao infinito na extremidades, e assim a partícula não conseguiria sair dessa região e ficaria presa. 
Para determinar o estado de uma partícula, usamos a equação de Schrödinger:

$$
-\frac{\hbar^{2} \nabla^{2}}{2 m} \psi(x, t)+\hat{V} \psi(x, t)=\hat{E} \psi(x, t) .
$$

Como o potencial dentro da região de confinamento é zero, podemos simplificar a equação da seguinte maneira:

$$
-\frac{\hbar^{2} \nabla^{2}}{2 m} \psi(x, t)=\hat{E} \psi(x, t) .
$$

A solução para essa equação é:

$$
\psi(x, t)=\operatorname{sen}\left(\frac{n \pi x}{l}\right) e^{-\frac{i E_{n} t}{h}}
$$

Podemos aplicar a equação (15) na equação (14), teremos:

$$
\operatorname{Esen}\left(\frac{n \pi x}{l}\right) e^{-\frac{i E_{n} t}{h}}=-\frac{\hbar^{2}}{2 m} \frac{\partial^{2} \operatorname{sen}\left(\frac{n \pi x}{l}\right) e^{-\frac{i E_{n} t}{h}}}{\partial x^{2}} .
$$

Fazendo as duas derivadas na função de onda, ficaremos com a energia total do sistema igual:

$$
E=\frac{n^{2} \pi^{2} \hbar^{2}}{2 m l^{2}}
$$

Agora vamos normalizar a função $\psi$ :

$$
\int_{0}^{l} \psi \psi^{*} d x=1
$$

sendo $\psi^{*}$ o complexo conjugado de $\psi$.

$$
\int_{0}^{l}\left(A \operatorname{sen}\left(\frac{n \pi x}{l}\right) e^{-\frac{i E_{n} t}{h}}\right)\left(A \operatorname{sen}\left(\frac{n \pi x}{l}\right) e^{+\frac{i E_{n} t}{h}}\right) d x=1 .
$$

Simplificando e resolvendo a integral teremos:

$$
A^{2}\left[\frac{x}{2}-\frac{1}{2} \frac{l}{2 n \pi} \operatorname{sen}\left(\frac{2 n \pi x}{l}\right)\right]_{0}^{l}=1 .
$$

Aplicamos os limites de integração e isolando a constante, Teremos que,

$$
A=\sqrt{\frac{2}{l}}
$$

A função de onda normalizada será:

$$
\psi(x, t)=\sqrt{\frac{2}{l}} \operatorname{sen}\left(\frac{n \pi x}{l}\right) e^{-\frac{i E_{n} t}{h}} .
$$

Até agora só foi trabalhado uma dimensão, falta agora em duas dimensões. Utilizando o mesmo caminho para achar a energia total da partícula confinada em uma dimensão:

$$
-\frac{\hbar^{2} \nabla^{2}}{2 m} \psi(x, t)=\hat{E} \psi(x, t) .
$$

Agora que estamos trabalhando com duas dimensões, a solução da equação de onda será:

$$
\psi(x, y, t)=\sqrt{\frac{2}{a}} \operatorname{sen}\left(\frac{n \pi x}{a}\right) \sqrt{\frac{2}{b}} \operatorname{sen}\left(\frac{n \pi y}{b}\right) e^{-\frac{i E_{n} t}{h}} .
$$

Sendo a e b as dimensões do plano onde está confinada a partícula. 
Aplicando a função de onda dada por (26) na equação (14), teremos:

$$
\begin{aligned}
& E \sqrt{\frac{2}{b}} \sqrt{\frac{2}{a}} \operatorname{sen}\left(\frac{n_{x} \pi x}{a}\right) \operatorname{sen}\left(\frac{n_{y} \pi y}{b}\right) e^{-\frac{i E_{n} t}{h}}= \\
& -\frac{\hbar^{2}}{2 m} e^{-\frac{i E_{n} t}{h}} \sqrt{\frac{2}{b}} \sqrt{\frac{2}{a}}\left[\frac{\operatorname{sen}\left(\frac{n_{x} \pi y}{b}\right) \partial^{2}\left(\operatorname{sen}\left(\frac{n \pi x}{l}\right)\right)}{\partial x^{2}}+\frac{\operatorname{sen}\left(\frac{n_{y} \pi x}{l}\right) \partial^{2}\left(\operatorname{sen}\left(\frac{n \pi y}{b}\right)\right)}{\partial y^{2}}\right] .
\end{aligned}
$$

Fazendo as derivas e as devidas simplificações, ficaremos um valor de energia igual a:

$$
E=\frac{\pi^{2} \hbar^{2}}{2 m}\left(\frac{n_{x}^{2}}{a^{2}}+\frac{n_{y}^{2}}{b^{2}}\right) .
$$

Na Mecânica Quântica o conceito de simetria é diferente. Temos um operador de simetria associado a um operador unitário. Se o operador hamiltoniano, que fornece informações sobre a energia do sistema quântico for invariante sobre a transformação:

$$
S^{+} H S=H,
$$

que é equivalente a termos o comutador entre $[\mathrm{G}, \mathrm{H}]=0$, na qual $\mathrm{G}$ é o gerador hermitiano do operador da simetria envolvida.

$$
S^{+}=1-\frac{i \epsilon}{\hbar} G
$$

Através da equação de movimento de Heisenberg, esse gerador é uma constante de movimento. Na Mecânica quântica teremos simetria ditas discretas e simetrias contínuas. Um desses operadores de simetria é o operador Paridade, que é mais simples de ser entendido. $\mathrm{O}$ operador Paridade ao atuar na função de onda promove uma espacial. Ou seja, faz x ir em -x. Podemos verificar o comportamento de outros operadores sob o ponto de vista da Paridade, como exemplo da simetria discreta em uma translação ao longo de uma rede cristalina.

\section{CONSIDERAÇÕES FINAIS}

Vimos que a simetria presente na Física Clássica tem sua forma alterada na Física Quântica. Estudamos as simetrias presentes na Física Clássica, a simetria associada a homogeneidade do espaço, a da isotropia do espaço. Na descrição quântica de um sistema físico, a sua função de onda fornece todas as informações necessárias para o seu entendimento, de acordo com os operadores associados às grandezas físicas de interesse. Estudamos os postulados da Mecânica Quântica, bem como a natureza dos Defeitos Topológicos presentes em um sólido cristalino. Vimos que esses defeitos estão associados às quebras de simetrias presentes no meio material.

\section{REFERÊNCIAS}

WEBBER, M. C. M.; RICCI, T. F., Inserção de mecânica quântica no ensino médio: uma proposta para professores. 2007. $63 \mathrm{f}$.

BOWMAN, G. Essential Quantum Mechanics. Northern Arizona University: Oxford, 2008.

KITTEL, C. Introduction to Solid State Physics. 8. ed, Wiley 2005.

HILL, C. T.; LEDERMAN, L. M.. Teaching symmetry in the introductory physics curriculum. The Physics Teacher, v. 38, n. 6, p.348-353, set. 2000.

GREINER, W.; MÜLLER, B. Quantum Mechanics: Symmetries. 2. Ed. Springer, 1989. 